\title{
Strong Isospin Breaking in Heavy-Meson Decay Constants: Employing Borelized QCD Sum Rules in Local-Duality Limit
}

\author{
Wolfgang Lucha ${ }^{1, *}$, Dmitri Melikhov ${ }^{1,2,3, * *}$, and Silvano Simula ${ }^{4, * * *}$ \\ ${ }^{1}$ Institute for High Energy Physics, Austrian Academy of Sciences, Nikolsdorfergasse 18, \\ A-1050 Vienna, Austria \\ ${ }^{2}$ D. V. Skobeltsyn Institute of Nuclear Physics, M. V. Lomonosov Moscow State University, \\ 119991 Moscow, Russia \\ ${ }^{3}$ Faculty of Physics, University of Vienna, Boltzmanngasse 5, A-1090 Vienna, Austria \\ ${ }^{4}$ INFN, Sezione di Roma Tre, Via della Vasca Navale 84, I-00146 Roma, Italy
}

\begin{abstract}
Applying the QCD sum-rule machinery in the so-called local-duality shape to heavy-light mesons reveals that, as a consequence of the non-zero mass gap between up and down quarks, the leptonic decay constants of the neutral and charged versions of the $D, D^{*}, B$ and $B^{*}$ mesons differ by approximately $1 \mathrm{MeV}$.
\end{abstract}

\section{Target: Heavy-Pseudoscalar- and -Vector-Meson Decay Constants}

The discrepancy $\left(m_{d}-m_{u}\right)(2 \mathrm{GeV}) \approx 2.5 \mathrm{MeV}[1]$ of the masses $m_{u, d}$ of up and down quarks is rather small compared to even the lightest meson masses but causes a strong-isospin-breaking disparity of the leptonic decay constants of mesons comprising heavy quark plus $u$ or $d$ quark.

We study pseudoscalar mesons $P_{q}$ and vector mesons $V_{q}$, subsumed by $H_{q}$, as heavy-light mesons formed by a heavy quark $Q=c, b$ of mass $m_{Q}$ and a light quark $q=u, d$, sof mass $m_{q}$, characterized by their masses $M_{H_{q}}=M_{P_{q}}, M_{V_{q}}$, four-momenta $p$, polarization vectors $\varepsilon_{\mu}(p)$ in the case of vector mesons $V_{q}$, and leptonic decay constants $f_{H_{q}}=f_{P_{q}}, f_{V_{q}}$, defined according to

$$
\left\langle 0\left|\bar{q}(0) \gamma_{\mu} \gamma_{5} Q(0)\right| P_{q}(p)\right\rangle=\mathrm{i} f_{P_{q}} p_{\mu}, \quad\left\langle 0\left|\bar{q}(0) \gamma_{\mu} Q(0)\right| V_{q}(p)\right\rangle=f_{V_{q}} M_{V_{q}} \varepsilon_{\mu}(p) .
$$

The formalism of QCD sum rules [2] provides an analytical approach to systems governed by the strong interactions. For our goals, it seems very promising to follow a path opened by a limiting case allowing us to concentrate all nonperturbative aspects within a single ingredient.

\section{Tool: (Borel-Transformed) QCD Sum Rule in its Local-Duality Limit}

A typical QCD sum rule [2] extracted from the two-point correlation function of appropriately chosen interpolating local operators relates basic properties of the hadron under consideration — in our case, of any heavy-light meson $H_{q}$ — to the parameters of QCD. The latter are either of fundamental nature, such as its strong fine-structure coupling $\alpha_{\mathrm{s}}$ and all quark masses, or of effective type, such as all the vacuum condensates $\langle\bar{q} q\rangle, \ldots$ encoding nonperturbative aspects.

\footnotetext{
*e-mail: Wolfgang.Lucha@oeaw.ac.at

**e-mail: dmitri_melikhov@gmx.de

***e-mail: simula@roma3.infn.it
} 
For convenience, in order to remove necessary subtraction terms and to suppress contributions of hadronic excitations and continuum, usually a Borel transformation from one's momentum variable to some Borel parameter, called $\tau$, is performed. The actual shape of such a QCD sum rule depends on its detailed formulation, reflected by a positive integer exponent $N=0,1, \ldots$ :

$$
\begin{aligned}
f_{H_{q}}^{2}\left(M_{H_{q}}^{2}\right)^{N} \exp \left(-M_{H_{q}}^{2} \tau\right) & =\int_{\left(m_{Q}+m_{q}\right)^{2}}^{s_{\mathrm{eff}}^{(N)}\left(\tau, m_{Q}, m_{q}, \alpha_{\mathrm{s}}\right)} \mathrm{d} s \exp (-s \tau) s^{N} \rho\left(s, m_{Q}, m_{q}, \alpha_{\mathrm{s}} \mid m_{\text {sea }}\right) \\
& +\prod_{\text {power }}^{(N)}\left(\tau, m_{Q}, m_{q}, \alpha_{\mathrm{s}},\langle\bar{q} q\rangle, \ldots\right) .
\end{aligned}
$$

On its QCD side, the three important ingredients are the $\tau$-dependent [3-7] effective threshold $s_{\text {eff }}^{(N)}\left(\tau, m_{Q}, m_{q}, \alpha_{\mathrm{s}}\right)$, constructed such as to guarantee mutual cancellation of perturbative-QCD and hadron contributions above the threshold to the utmost achievable degree; the perturbative spectral density $\rho\left(s, m_{Q}, m_{q}, \alpha_{\mathrm{s}} \mid m_{\text {sea }}\right)$, experiencing also the masses $m_{\text {sea }}$ of the sea quarks and expressible as an expansion (Fig. 1) in powers of $\alpha_{\mathrm{s}}$ depending on the renormalization scale $\mu$,

$$
\begin{aligned}
\rho\left(s, m_{Q}, m_{q}, \alpha_{\mathrm{s}} \mid m_{\mathrm{sea}}\right) & =\rho_{0}\left(s, m_{Q}, m_{q}\right)+\frac{\alpha_{\mathrm{s}}(\mu)}{\pi} \rho_{1}\left(s, m_{Q}, m_{q}, \mu\right) \\
& +\frac{\alpha_{\mathrm{s}}^{2}(\mu)}{\pi^{2}} \rho_{2}\left(s, m_{Q}, m_{q}, \mu \mid m_{\text {sea }}\right)+O\left(\alpha_{\mathrm{s}}^{3}\right)
\end{aligned}
$$

and the $\tau$-dependent power corrections $\Pi_{\text {power }}^{(N)}\left(\tau, m_{Q}, m_{q}, \alpha_{\mathrm{s}},\langle\bar{q} q\rangle, \ldots\right)$ that subsume, together with the effective threshold $s_{\text {eff }}^{(N)}\left(\tau, m_{Q}, m_{q}, \alpha_{\mathrm{s}}\right)$, all the nonperturbative manifestations of QCD.

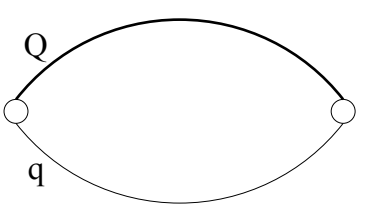

(a)

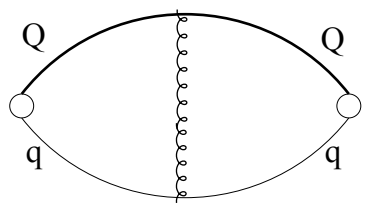

(b)

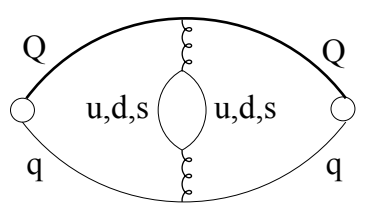

(c)

Figure 1. Perturbative expansion (2) of the spectral density $\rho\left(s, m_{Q}, m_{q}, \alpha_{\mathrm{s}} \mid m_{\text {sea }}\right)$ in powers of the strong coupling $\alpha_{\mathrm{s}}$ : (a) $O(1)$, (b) $O\left(\alpha_{\mathrm{s}}\right)$, (c) $O\left(\alpha_{\mathrm{s}}^{2}\right)$; sea quarks, and thus their masses, start to contribute at $O\left(\alpha_{\mathrm{s}}^{2}\right)$.

The partitioning of nonperturbative contributions between power corrections and effective threshold reflects, in general, the details of the problem to which the QCD sum rule is applied. By considering, however, the local-duality limit $\tau \rightarrow 0$ of Eq. (1) for the handpicked exponent $N=0$ such that all power corrections vanish we may achieve to shift all of the nonperturbative burden to the effective threshold. With all power corrections gone, Eq. (1) simplifies to [8-10]

$$
\left.f_{H_{q}}^{2}=\int_{\left(m_{Q}+m_{q}\right)^{2}}^{s_{\mathrm{eff}}} \mathrm{m} s \rho\left(s, m_{Q}, m_{q}, \alpha_{\mathrm{s}}\right) \alpha_{\mathrm{s}} \mid m_{\mathrm{sea}}\right) \equiv F\left(s_{\mathrm{eff}}\left(m_{q}\right), m_{q} \mid m_{\mathrm{sea}}\right) .
$$

All spectral densities required as input are known up to order $O\left(\alpha_{\mathrm{s}} m_{q}\right)$ and $O\left(\alpha_{\mathrm{s}}^{2} m_{q}^{0}\right)$ [11-14]. However, we first must ensure the well-definiteness of the local-duality limit $\tau \rightarrow 0$ of Eq. (1).

The dependence on the Borel parameter $\tau$ of our effective threshold $s_{\text {eff }}^{(N)}\left(\tau, m_{Q}, m_{q}, \alpha_{\mathrm{s}}\right)$ and of the power corrections $\Pi_{\text {power }}^{(N)}\left(\tau, m_{Q}, m_{q}, \alpha_{\mathrm{s}},\langle\bar{q} q\rangle, \ldots\right)$ under the "disguise" of some quantity

$$
\bar{\Pi}_{\text {power }}^{(N)}(\tau) \equiv \Pi_{\text {power }}^{(N)}(\tau) \frac{\exp \left(M_{H_{q}}^{2} \tau\right)}{M_{H_{q}}^{2 N}}
$$


is explored, for the three most interesting values $N=0,1,2$ of the exponent $N$, in Fig. 2 for the pseudoscalar mesons and in Fig. 3 for the vector mesons. For the cases $N=0$ and $N=1$, the $\tau$ behaviour of the power corrections at $\tau=0$ is regular: for $N=0$, all power corrections vanish,

$$
\lim _{\tau \rightarrow 0} \Pi_{\text {power }}^{(N)}\left(\tau, m_{Q}, m_{q}, \alpha_{\mathrm{s}},\langle\bar{q} q\rangle, \ldots\right)=0 \quad \text { for } \quad N=0 ;
$$

for $N=1$, the power corrections approach a finite value. In contrast to this, for the case $N=2$, due to an involvement of also $\log (\tau)$ terms the power corrections exhibit singularities at $\tau=0$. Hence, in our pursuit of local-duality QCD sum rules the exponent of choice must $\operatorname{read} N=0$.

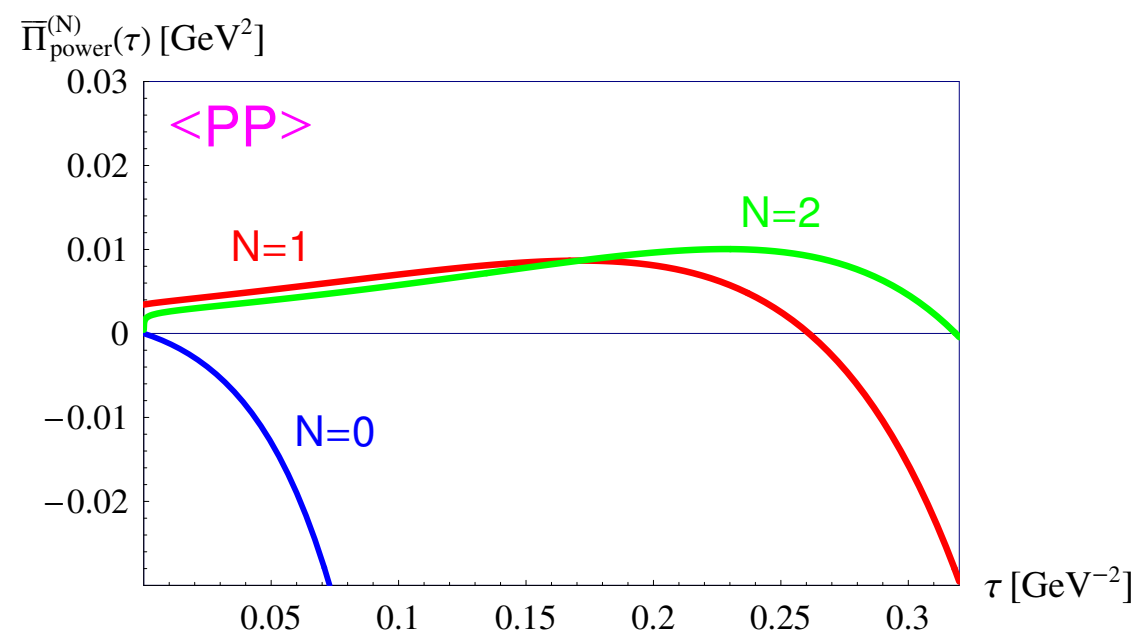

(a)

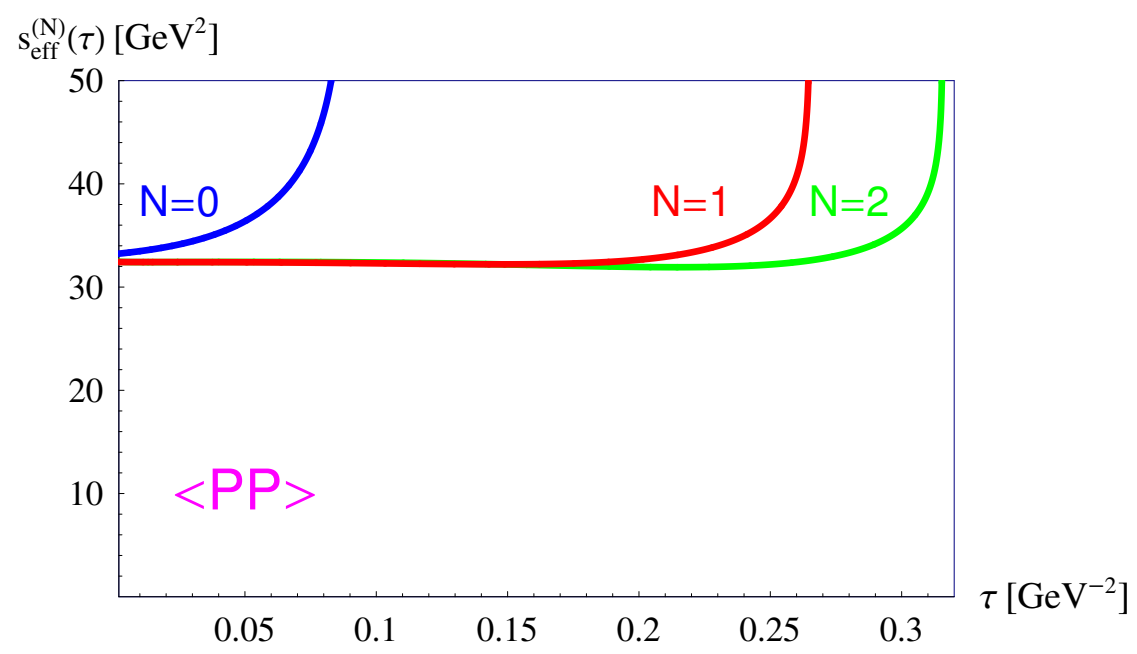

(b)

Figure 2. Dependence of (a) the power corrections $\Pi_{\text {power }}^{(N)}(\tau)$, rescaled according to Eq. (4), as well as (b) our effective threshold $s_{\text {eff }}^{(N)}(\tau)$ in the QCD sum rule for two pseudoscalar currents on the Borel variable $\tau$. 


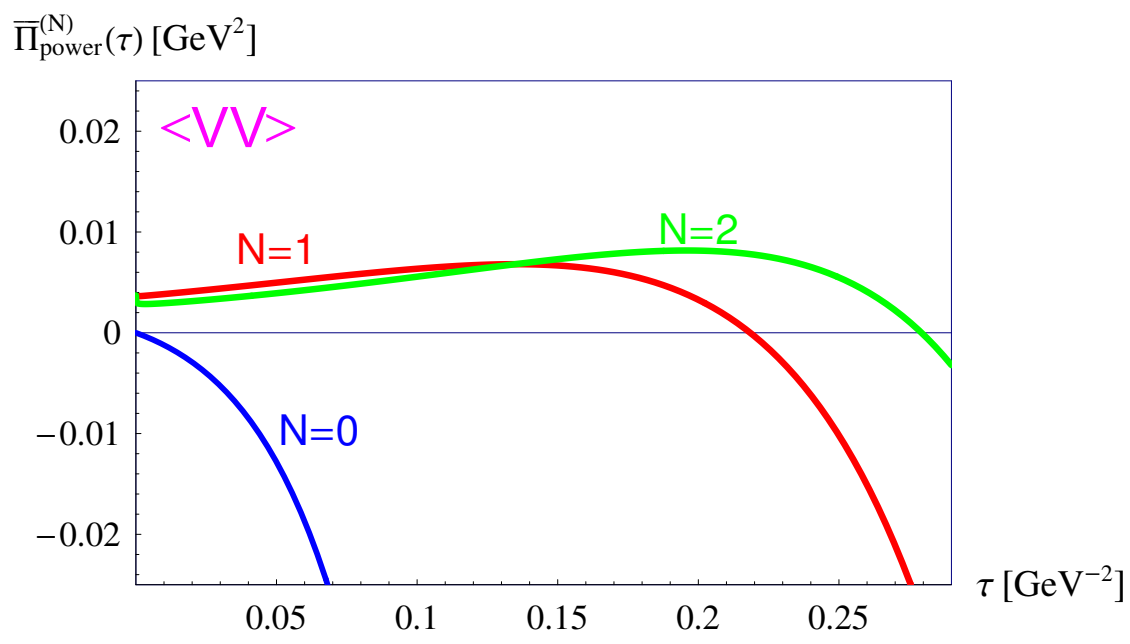

(a)

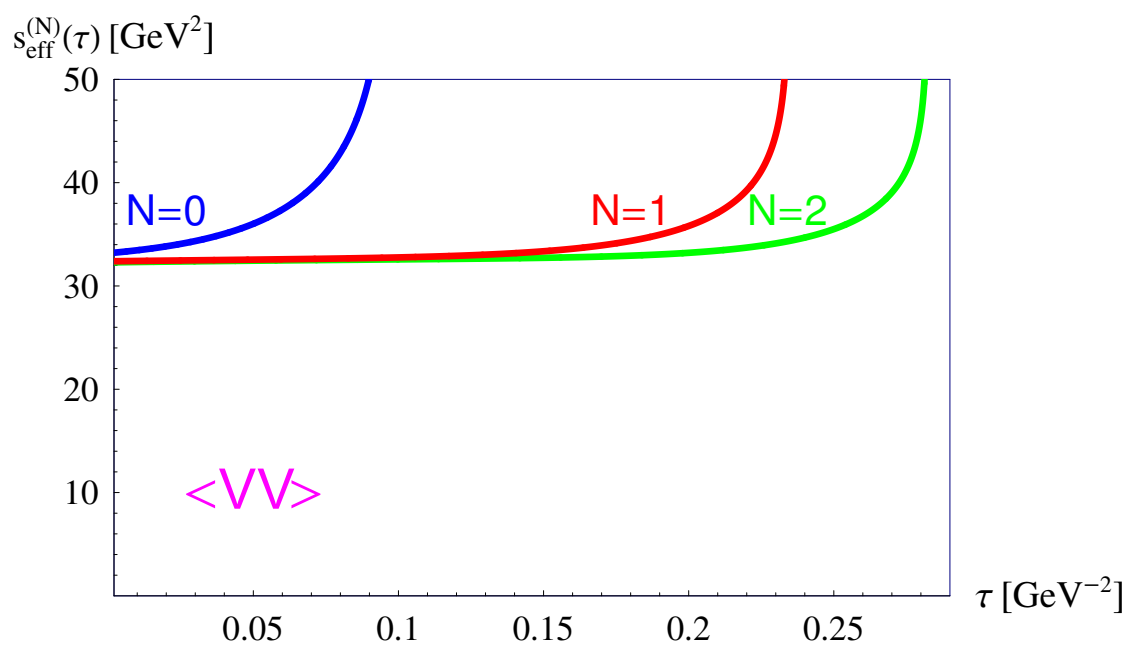

(b)

Figure 3. Dependence of (a) the power corrections $\Pi_{\text {power }}^{(N)}(\tau)$, suitably rescaled according to Eq. (4), and (b) our effective threshold $s_{\text {eff }}^{(N)}(\tau)$ in the QCD sum rule for two vector operators on the Borel parameter $\tau$.

\section{Triumph: Local-Duality Outcomes for Decay-Constant Differences}

By collecting the available pieces of information about the $m_{q}$ dependence of all the quantities entering in our local-duality limit (3) of the QCD sum rule (1), we extract the isospin-breaking difference $f_{H_{d}}-f_{H_{u}}$ from the difference of an associated function $F\left(s_{\text {eff }}\left(m_{q}\right), m_{q} \mid m_{\text {sea }}\right)$, Eq. (3):

$$
\begin{aligned}
& F\left(s_{\mathrm{eff}}\left(m_{d}\right), m_{d} \mid m_{\mathrm{sea}}\right)-F\left(s_{\mathrm{eff}}\left(m_{u}\right), m_{u} \mid m_{\mathrm{sea}}\right) \\
& =F\left(s_{\mathrm{eff}}\left(m_{d}\right), m_{d} \mid 0\right)-F\left(s_{\mathrm{eff}}\left(m_{u}\right), m_{u} \mid 0\right)+O\left(\alpha_{\mathrm{s}}^{2}\left(m_{d}-m_{u}\right)\right) .
\end{aligned}
$$



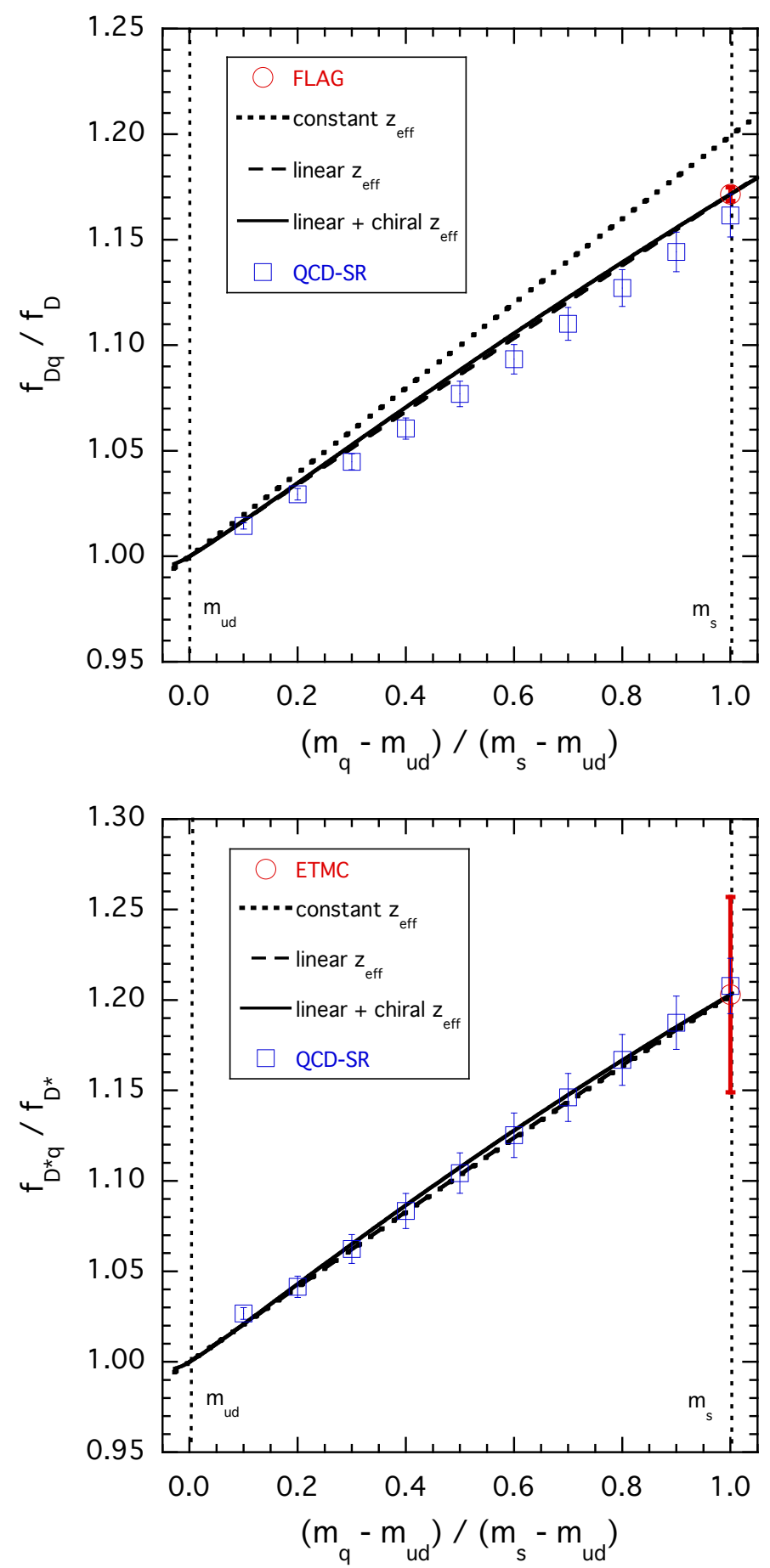

Figure 4. Dependence of the heavy-light charmed-meson decay constants, $f_{D_{q}^{(*)}} \equiv f_{D_{q}^{(*)}}\left(m_{q}\right)$, normalized to their values $f_{D^{(*)}} \equiv f_{D_{q}^{(*)}}\left(m_{u d}\right)$ at the average light-quark mass, $m_{u d} \equiv\left(m_{u}+m_{d}\right) / 2$, on the (conveniently shifted and rescaled) light-quark mass $\left(m_{q}-m_{u d}\right) /\left(m_{s}-m_{u d}\right)$, as inferred from three different assumptions about the behaviour of our effective threshold $z_{\text {eff }} \equiv \sqrt{s_{\text {eff }}}-m_{c}-m_{q}$ [8-10], vs. previous findings [15] (口). 

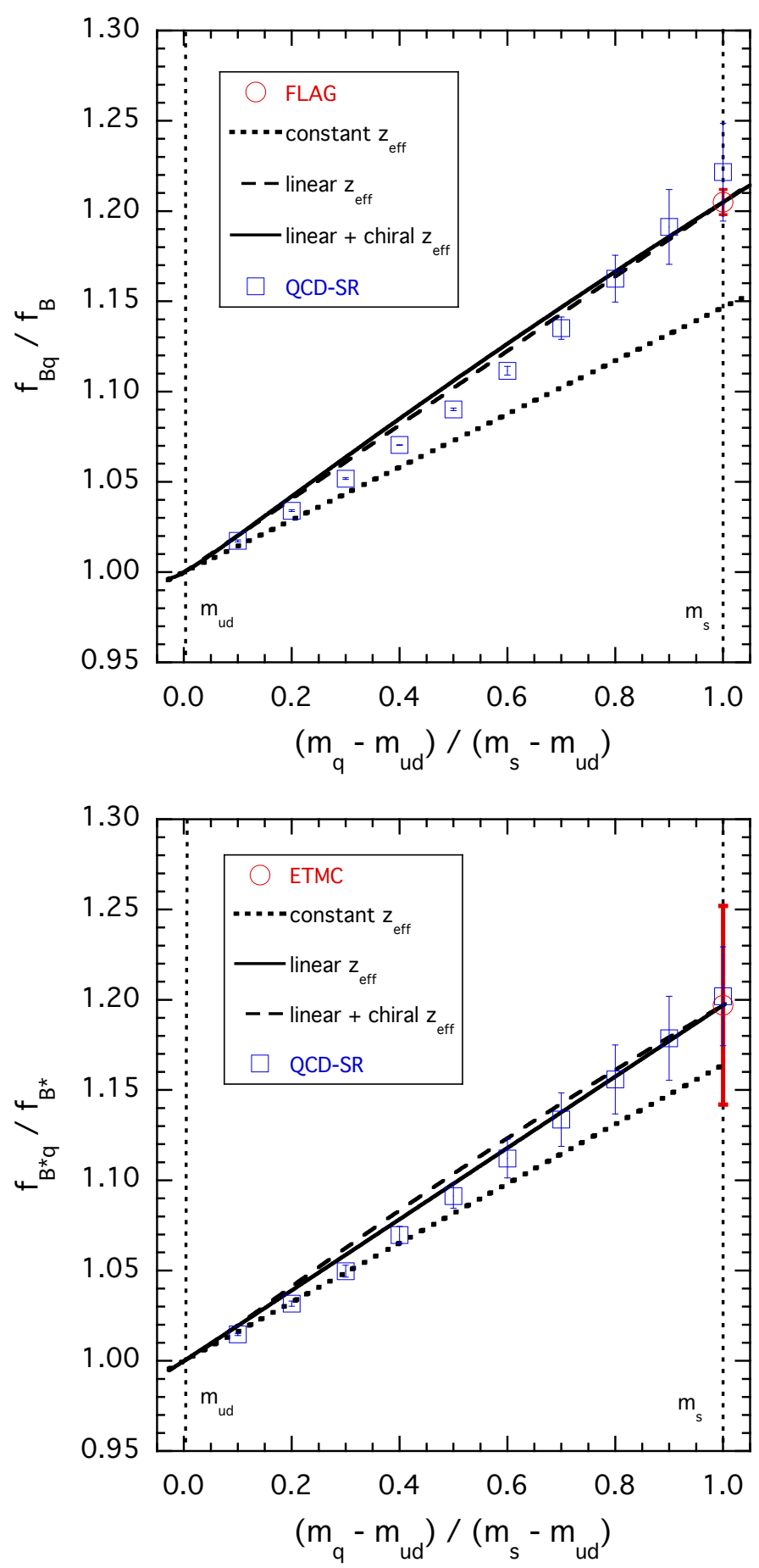

Figure 5. Dependence of the heavy-light bottom-meson decay constants $f_{B_{q}^{(*)}} \equiv f_{B_{q}^{(*)}}\left(m_{q}\right)$, normalized to their values $f_{B^{(*)}} \equiv f_{B_{q}^{(*)}}\left(m_{u d}\right)$ at the average light-quark mass, $m_{u d} \equiv\left(m_{u}+m_{d}\right) / 2$, on the - still suitably shifted and rescaled - light-quark mass $\left(m_{q}-m_{u d}\right) /\left(m_{s}-m_{u d}\right)$, inferred from three different assumptions about the behaviour of our effective threshold $z_{\mathrm{eff}} \equiv \sqrt{s_{\mathrm{eff}}}-m_{b}-m_{q}[8-10]$, vs. previous findings [15] (口). 
All dependence on the light-quark mass $m_{q}$ of our predictions for leptonic decay constants $f_{H_{q}}$ by the local-duality QCD sum rule (3) originates in precisely two sources of different type:

1. The impact of the spectral density can be trivially inferred from existing knowledge due to perturbation theory. Equation (5) already takes into account that in this difference the contributions of strange sea quarks cancel. This reduces the uncertainties considerably.

2. The situation with the effective threshold is more delicate: we model it by allowing for a continuous variation of $m_{q}$ within $\left[0, m_{s}\right]$ and matching all results for $f_{H_{q}}$ to lattice QCD in three neighbouring ways, see Fig. 4 for charmed quarks and Fig. 5 for bottom quarks.

In proper naming, our results for the heavy-light meson decay-constant differences are [8-10]

$$
\begin{array}{rlrl}
f_{D^{ \pm}}-f_{D^{0}} & =(0.96 \pm 0.09) \mathrm{MeV}, & & f_{D^{* \pm}}-f_{D^{* 0}}=(1.18 \pm 0.35) \mathrm{MeV}, \\
f_{B^{0}}-f_{B^{ \pm}} & =(1.01 \pm 0.10) \mathrm{MeV}, & f_{B^{* 0}}-f_{B^{* \pm}}=(0.89 \pm 0.30) \mathrm{MeV} .
\end{array}
$$

Strong isospin breaking in these heavy-light-meson decay constants is pretty close to $1 \mathrm{MeV}$.

\section{Acknowledgements}

D. M. is grateful for support by the Austrian Science Fund (FWF) under project P29028-N27.

\section{References}

[1] Particle Data Group (M. Tanabashi et al.), Phys. Rev. D 98 (2018) 030001.

[2] M. A. Shifman, A. I. Vainshtein, and V. I. Zakharov, Nucl. Phys. B 147 (1979) 385.

[3] W. Lucha, D. Melikhov, and S. Simula, Phys. Rev. D 79 (2009) 096011, arXiv:0902. 4202 [hep-ph].

[4] W. Lucha, D. Melikhov, and S. Simula, J. Phys. G 37 (2010) 035003, arXiv:0905.0963 [hep-ph].

[5] W. Lucha, D. Melikhov, H. Sazdjian, and S. Simula, Phys. Rev. D 80 (2009) 114028, arXiv:0910.3164 [hep-ph].

[6] W. Lucha, D. Melikhov, and S. Simula, Phys. Lett. B 687 (2010) 48, arXiv:0912.5017 [hep-ph].

[7] W. Lucha, D. Melikhov, and S. Simula, Phys. Atom. Nucl. 73 (2010) 1770, arXiv:1003. 1463 [hep-ph].

[8] W. Lucha, D. Melikhov, and S. Simula, Eur. Phys. J. C 78 (2018) 168, arXiv:1702.07537 [hep-ph].

[9] W. Lucha, D. Melikhov, and S. Simula, PoS (EPS-HEP 2017) 669, arXiv:1709.02131 [hep-ph].

[10] W. Lucha, D. Melikhov, and S. Simula, PoS (Hadron2017) 175, arXiv:1711.07899 [hep$\mathrm{ph}]$.

[11] K. G. Chetyrkin and M. Steinhauser, Phys. Lett. B 502 (2001) 104, arXiv:hep-ph/ 0012002.

[12] K. G. Chetyrkin and M. Steinhauser, Eur. Phys. J. C 21 (2001) 319, arXiv:hep-ph/ 0108017.

[13] M. Jamin and B. O. Lange, Phys. Rev. D 65 (2002) 056005, arXiv:hep-ph/0108135.

[14] P. Gelhausen, A. Khodjamirian, A. A. Pivovarov, and D. Rosenthal, Phys. Rev. D 88 (2013) 014015, arXiv:1305.5432 [hep-ph]; Phys. Rev. D 89 (2014) 099901(E); 91 (2015) 099901(E).

[15] W. Lucha, D. Melikhov, and S. Simula, Phys. Lett. B 765 (2017) 365, arXiv:1609.05050 [hep-ph]. 